\title{
Vocalization-related stapedius muscle activity in different age chickens (Gallus gallus), and its role in vocal development
}

\author{
Silvarosa Grassi ${ }^{1}$, Fabrizio Ottaviani ${ }^{2}$ and Domenico Bambagioni ${ }^{1}$ \\ ${ }^{1}$ Institute of Human Physiology, University of Perugia, Perugia (Italy) and ${ }^{2}$ Institute of Otorhinolaryngology, Catholic University, \\ Rome (Italy)
}

(Accepted 3 April 1990)

Key words: Stapedius muscle; Vocalization; Electrical stapedius muscle response; Vocal development; Power spectrum; Auditory feedback

\begin{abstract}
The stapedius muscle activity associated with vocalization was analyzed in young and adult roosters. Our results show that remarkable differences in the behavior of vocalization-related stapedius muscle activity exist between these two ages. Unlike young roosters, electrical stimulation in the midbrain of adult cocks yields vocalizations associated with stapedius muscle EMG responses that always show a higher threshold and a longer latency than those of the vocalization induced. Moreover, the maximal amplitude of the stapedius muscle EMG response is consistently lower than that detected in young roosters, despite the fact that the maximal vocalization amplitude of the adult birds is much higher. On the whole our results demonstrate that vocalization-related stapedius muscle activity is strongly reduced in adulthood. The possibility that stapedius muscle may play a role during the vocal development was verified by comparing the crow of normal roosters with that of cocks from which the stapedius muscle had been removed shortly after hatching. Strong differences exist in the amplitude/frequency distribution of the crowing of normal and stapedectomized roosters, suggesting that the stapedius muscle exerts an important role in auditory feedback modulation, and that this feedback is used for normal vocal development.
\end{abstract}

\section{INTRODUCTION}

It is known that, unlike mammals, chickens (as other avian species) do not show an acoustic stapedial reflex ${ }^{1}$, 4,16 , while the stapedius muscle is activated during the animal's own vocalization, causing a change of the acoustic impedance of the ear ${ }^{1-6}$.

Stapedius activity associated with vocalization in chickens occurs in response to a central stimulus that reaches the mesencephalic stapedius controlling neurons and is produced by neurons involved in vocalization ${ }^{8,9}$.

Despite some hypotheses, the physiological significance of vocalization-related stapedius muscle activity in chickens has not been fully clarified. It has been proposed that it may: (1) insure normal 'auditory vigilance' during vocalization; and (2) 'protect' the inner ear receptor cells against overstimulation by the bird's own voice ${ }^{1,4,5}$.

Since the variables affecting vocal development in birds have not been fully described, a possible involvement of stapedius muscle activity in this development seems possible.

Comparative studies of different species show that the course of vocal development is inflexible in some birds and plastic in others ${ }^{11,12}$. The domestic chicken is considered to belong to the former group, because the basic pattern of its calls and crowing as they appear on audiospectrograms is not affected by early deafening ${ }^{10}$.

By contrast, it has been suggested that songbirds can develop a normal vocalization only by hearing themselves vocalize, using acoustic feedback control of voice ${ }^{11-15}$.

Our personal unpublished observation that stapedius muscle size is reduced in adult roosters, compared with that of young ones, induced us to begin the present study aimed at verifying whether stapedius muscle, activated only by the cock's own voice, might play a role during vocal development. This work was carried out first by analyzing the vocalization-related stapedius muscle activity at different stages of development and then by comparing the crow (considered the most elaborate vocalization) of normal roosters with that of cocks from which the stapedius muscle had been removed shortly after hatching.

\section{MATERIALS AND METHODS}

Vocalization-related stapedius muscle activity in chickens of different age

The experiments were performed on 20 male Leghorn chickens (Gallus gallus) divided into two groups: one made up of 10 young (40-60 days old) roosters (group A) and the other of 10 adult (130-150 days old) ones (group B).

Group $A$ roosters, weighed $1.5-1.8 \mathrm{~kg}$ and were characterized by juvenile secondary sexual characters (cockscomb and wattles) and

Correspondence: S. Grassi, Istituto di Fisiologia Umana, Università di Perugia, Via del Giochetto, I-06100 Perugia, Italy. 
by an immature vocalization. Group B roosters, weighing $3.0-3.5 \mathrm{~kg}$ showed mature secondary sexual characters and crowed.

In both young and adult cocks, a stapedius muscle electromyogram (EMG) was recorded under urethane anesthesia $(1 \mathrm{~g} / \mathrm{kg} \mathrm{b}$. wt., i.v.) during vocalization elicited by electrical stimulation of the mesencephalic 'calling area' according to Grassi et al. ${ }^{7-9}$.

Stapedius muscle response alone, elicited by stimulating the mesencephalic stapedius controlling area, was also analyzed. The sites stimulated were tested by increasing stimulus intensities (from 0 to $100 \mu \mathrm{A}$ ), square pulses $0.5 \mathrm{~ms}$ duration delivered at a rate of 100 pulses $/ \mathrm{s}^{17}$.

In adult cocks a stapedius muscle electromyogram (EMG) was also recorded during vocalization elicited by electrical stimulation of the occipitomesencephalicus tract rostral to the midbrain ${ }^{17}$. Vocalization was recorded using a microphone placed at a distance of about $7 \mathrm{~cm}$ from the cock's beak (thus delaying the response by about $0.2 \mathrm{~ms}$ ) and stapedius muscle EMG response was recorded by means of a bipolar electrode consisting of two $0.1 \mathrm{~mm}$ copper wires, insulated except at the tip. Electrical stapedius muscle response (EMG, filtered between 100 and $10000 \mathrm{~Hz}$ ) and vocalization $(300-3000 \mathrm{~Hz})$ were recorded on a digital storage oscilloscope, transferred to a computer and stored on diskettes.

The amplitude of both EMG and vocalization responses was evaluated by measuring the area (integration) of the full-wave rectified EMG and of vocalization activity. The localization of the stimulating electrode in the midbrain, was subsequently verified after appropriate histological processing of the brain. For further technical details see Grassi et al. ${ }^{8,9}$.

\section{Stapedius muscle and vocalization development}

In order to verify whether the stapedius muscle was involved in vocal development, 15 male Leghorn chickens were used, all born on the same day, on the same chicken farm. Five chicks underwent bilateral stapedius muscle removal at 10 days of age. Five chicks underwent a sham operation and the others served as controls. Experimental animals were anesthetized with ether by inhalation, and the stapedius muscle was exposed and completely detached from its origin along the temporal and occipital bones, and cut at the level of its entrance into the middle ear cavity. During this procedure the tympanic cavity was not at all opened or damaged. For sham operations, all surgical procedure, except the muscle removal, was performed. Both experimental and control chicks were handled equally, and maintained in identical social conditions for equal lengths of time.

Testing of vocal behavior was performed in operated and control cocks every 20 days from birth until the appearance of crowing at about 100-120 days of age. Crows were recorded until their pattern became stable. Sound recordings (at $9.5 \mathrm{~cm} / \mathrm{s}$ speed) were low-pass filtered $(5000 \mathrm{~Hz})$ and digitalized at $10000 \mathrm{~Hz}$ through an IBM-XT computer equipped with Computerscope ISC-16 System (R.C. Electronics, Inc.) at $0.1 \mathrm{~ms}$ sampling rate.

The frequency response of the tape recorder was $\pm 3 \mathrm{~dB}$ in the range $200-10000 \mathrm{~Hz}$. Each crow, lasting $1.8-2.2 \mathrm{~s}$, was analyzed by a program (I.L.S., Signal Technology, Inc.) which computed the average power spectrum based on Fast Fourier Transform (FFT) of 256 points time series $(25.6 \mathrm{~ms})$. The amplitude value for each spectral peak was expressed as percentage of the total energy of that spectrum. The amplitudes of crow components of normal and operated roosters were statistically compared by using the MannWhitney test.

\section{RESULTS}

\section{Vocalization-related stapedius muscle activity in young and adult roosters}

As previously reported ${ }^{8,9}$ electrical stimulation of the mesencephalic 'calling area' of Gallus gallus consistently elicited stapedius muscle activity, which appeared to be associated with vocalization both in young and adult roosters. In young roosters (group A), in full agreement with previous results ${ }^{9}$, stapedius muscle EMG response showed the same or a slightly higher threshold than that of vocalization, depending on the exact locus stimulated within the mesencephalic 'calling area' and its latency changed according to a direct or secondary activation of the mesencephalic stapedius controlling neurons ${ }^{9}$. The amplitude of both responses increased with the stimulus intensity until a maximal response was elicited (Fig. 1A).

In adult roosters (group B) stimulation of any point within the mesencephalic 'calling area', elicited stapedius muscle EMG at a threshold much higher than that of vocalization (Fig. 1B). In fact stapedius muscle EMG response appeared only at stimulus intensities evoking loud vocalizations and its latency was constantly longer than that of vocalization, independently of stimulus intensities. Moreover in group B roosters, the amplitude of vocalization response strongly increased with increasing stimulus strength, but the EMG response amplitude rose little and the maximal EMG response was always much lower than that observed in group A cocks. This effect is illustrated in the histogram in Fig. 2, showing a reduction of the vocalization-evoked stapedius muscle activity in adult roosters compared to young ones.

Stapedius muscle EMG response alone, elicited by stimulating the mesencephalic stapedius controlling area, was also analyzed in both groups. In group $A$ roosters the threshold for stapedius muscle EMG response was lower $(12 \pm 1.3 \mu \mathrm{A})$ than that detected in adult ones $(72 \pm 3.7$ $\mu \mathrm{A})$. Also in this case, the maximal response recorded in adult roosters was always lower than that elicited in young ones (Fig. 3). This result was followed by the finding, in group B roosters, of a reduction in the size of the mesencephalic locus, from which stimulation elicited stapedius muscle response independent of vocalization (Fig. 4). This area, located extensively around the mesencephalic 'calling area' in young roosters', was confined to a narrow mesencephalic locus in adult animals, where, on the contrary, an enlargement of the 'calling area' could be detected (Fig. 4).

Stimulation at a more anterior level (A 10.00, L 3.00, H 5.00) ${ }^{6}$, corresponding to tractus occipitomesencephalicus, was also performed in adult roosters to rule out the possibility that a higher stapedius muscle activity could be associated with the activation of supramesencephalic loci. In full agreement with previous results stimulation of these higher loci evoked vocalization responses like those evoked by stimulating the mesencephalic 'calling area'; these vocal responses were associated with a low stapedius muscle EMG response perfectly superimposable on 
A
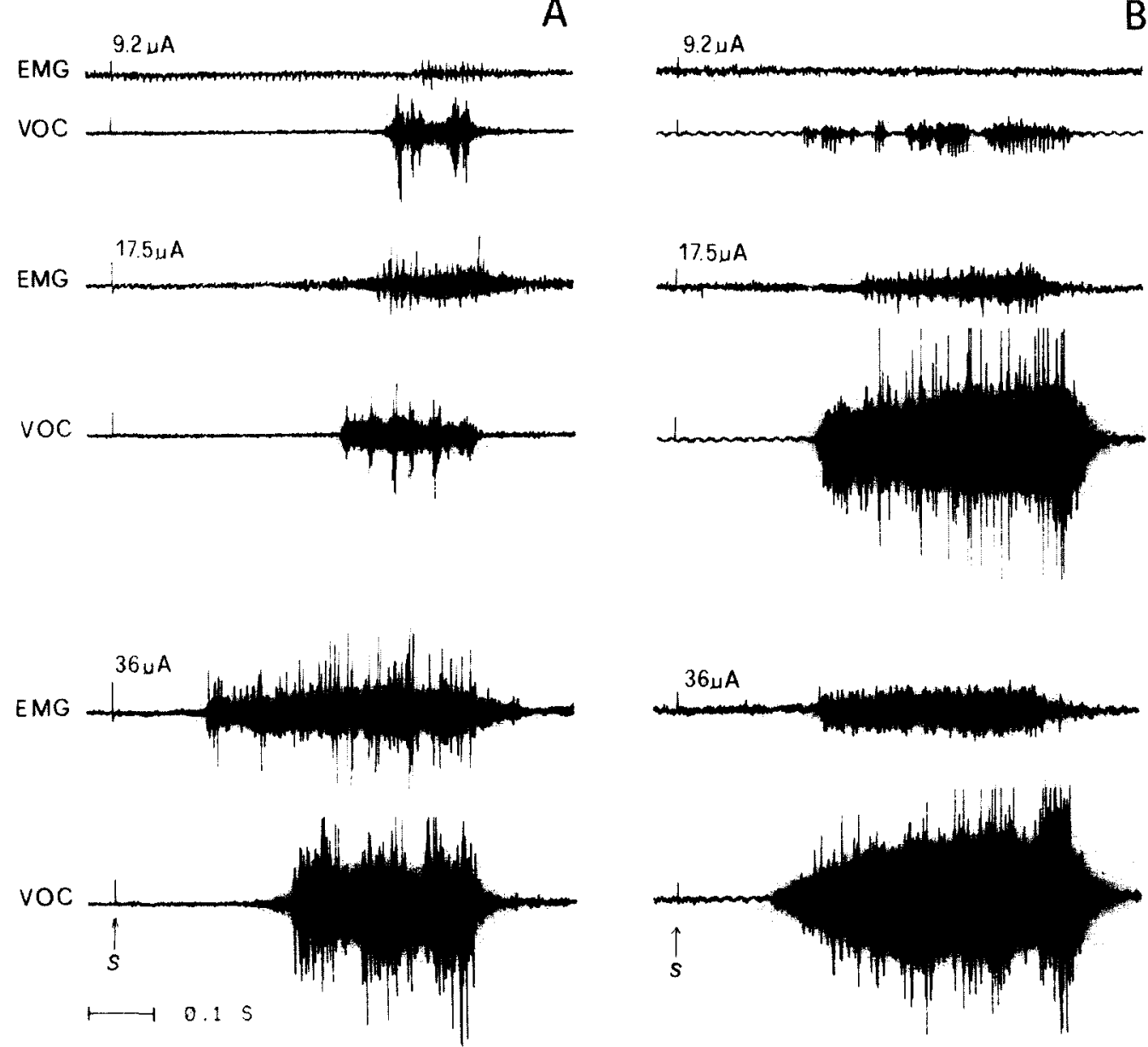

Fig. 1. Stapedius muscle EMG response (EMG) and vocalization (VOC) elicited by stimulating the low threshold mesencephalic vocalization loci in young $(\mathrm{A})$ and adult $(\mathrm{B})$ roosters. Stimulus intensities $(\mu \mathrm{A})$ are indicated on the left of each recording and the beginning of the stimulation by $\mathrm{S}$. Time calibration applies to all recordings.

that elicited by stimulating the mesencephalic 'calling area' (Figs. 5 and 6).

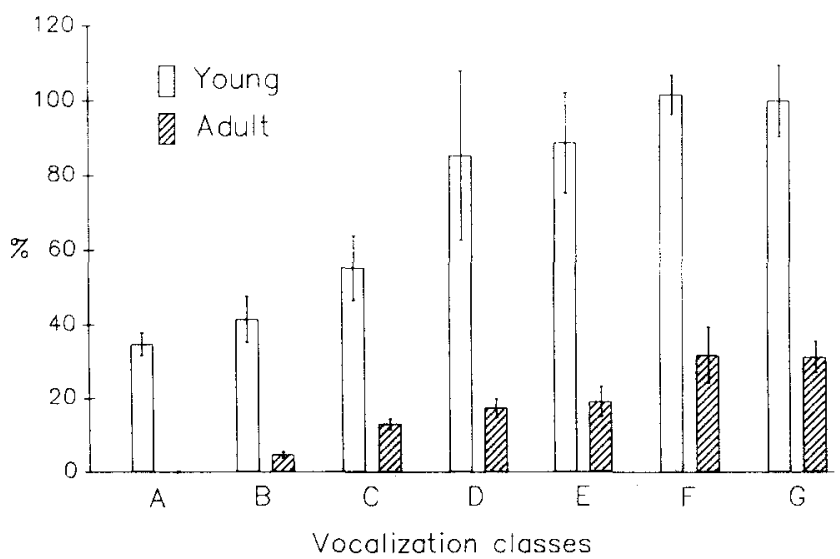

Fig. 2. EMG amplitude as a function of vocalization amplitude, expressed as classes of percentage of the maximal vocalization amplitude obtained in young and adult roosters $(\mathrm{A}, 0-10 \%$; $\mathrm{B}$, $10-20 \%$; C, $20-30 \%$; D, 30-40\%; E, 40-50\%; F, 50-60\%; and G, $60-100 \%$ ). The amplitude values are the area of full-wave rectified EMG and of vocalization activity. Mean of the recordings obtained in 10 fowl \pm S.E.M.
On the whole these results suggest that vocalizationrelated stapedius muscle activity is strongly reduced in adulthood.

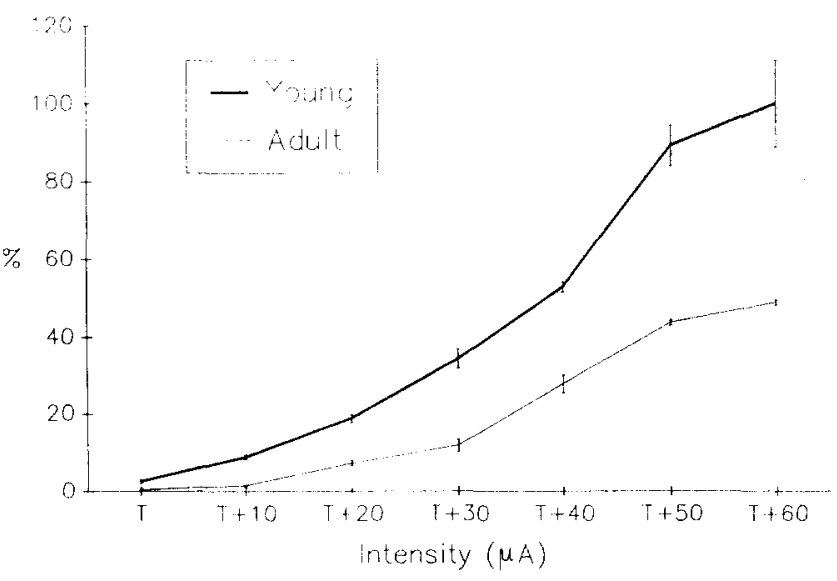

Fig. 3. Plot of the amplitude of EMG elicited without vocalization (percentage of the maximum, ordinate) as a function of stimulus intensity above threshold ( $\mathrm{T}$ abscissa) in young and adult roosters. The EMG amplitude values are the area of full-wave rectified EMG activity. Each point is the mean of the responses obtained in 10 fowl \pm S.E.M 
A

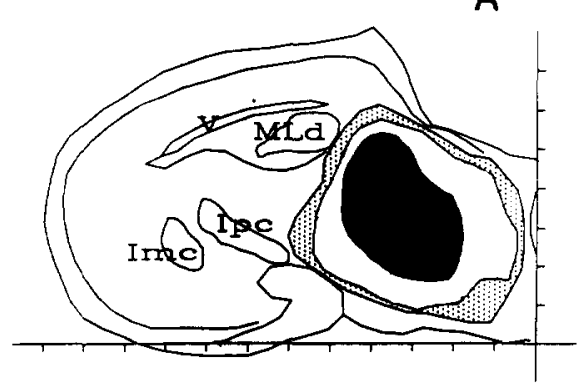

B

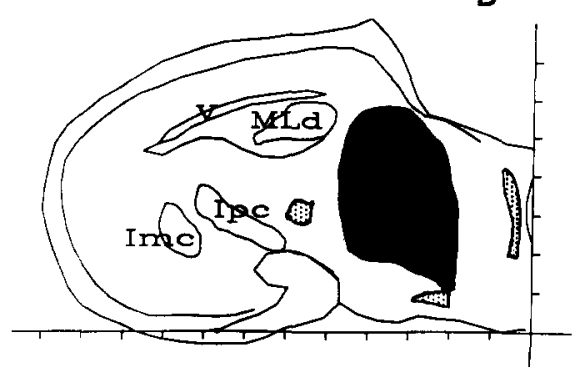

Fig. 4. Mesencephalic areas from which vocalization response (black area) and stapedius muscle activity alone (dotted area) could be elicited in young (A) and adult (B) roosters are plotted on a schematic section of the chicken brain stereotaxic atlas ${ }^{7}$. On both axes, each division represents $1 \mathrm{~mm}$. Imc, nucleus isthmi, pars magnocellularis; Ipc, nucleus isthmi, pars parvocellularis; MLd, nucleus mesencephalicus lateralis, pars dorsalis; V, ventriculus.

$26 \mu \mathrm{A}$

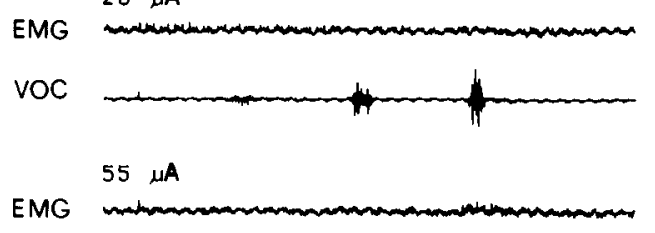

VOC

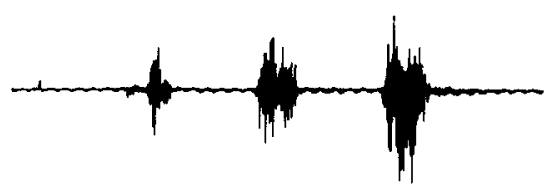

$100 \mu \mathrm{A}$
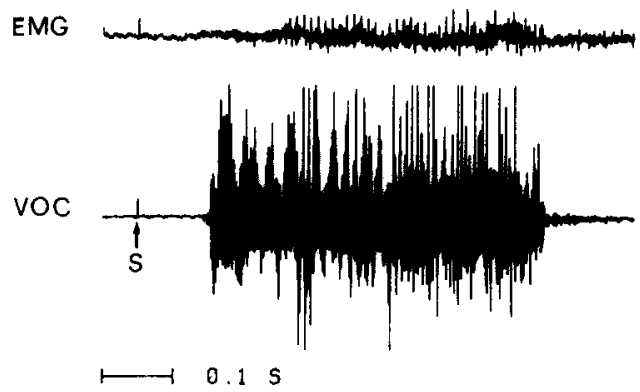

Fig. 5. Stapedius muscle EMG response (EMG) and vocalization (VOC) elicited in adult roosters by stimulating tractus occipitomesencephalicus at supramesencephalic level. Stimulus intensities $(\mu \mathrm{A})$ are indicated on the left of each recording and the beginning of the stimulation by $\mathrm{S}$. Time calibration applies to all recordings.
Acoustic structure of calls in normal and stapedectomized adult roosters

Both operated, sham operated and normal roosters began to crow at about 100-120 days, after a progressive change of their vocalization, characterized by a shifting of vocal acoustic energy from higher frequencies (2000$4000 \mathrm{~Hz})$, typical of the chick, to lower ones $(400-2000$ $\mathrm{Hz}$ ), typical of the adult cocks.

The removal of the stapedius muscle did not affect the volume of crowing and its temporal pattern. In fact, the total energy value of each crow and the crow duration (1.8-2.2 s) were similar in both normal, sham operated and operated roosters. Furthermore, both roosters crowed with the same repetition rate during the day.

On the contrary, significant differences in the energy content of various crow components existed between normal and stapedectomized roosters. Although slight differences could be already detected in vocalization at about 60-80 days of life, they were particularly evident in the crowing, and progressively increased from the time the crow appeared until its pattern became stable (Fig. 7).

In fact, the power spectrum of crow showed the acoustic energy distributed in two main peaks, at $633 \pm$ $31 \mathrm{~Hz}$ and $1300 \pm 81 \mathrm{~Hz}$ in the normal and sham operated roosters, and at $700 \pm 17 \mathrm{~Hz}$ and $1400 \pm 56 \mathrm{~Hz}$ in the operated ones (Fig. 7). The differences in the peak frequencies between normal and operated roosters were not statistically significant $(P>0.05)$. However, the energy content of each peak was clearly different between operated and unoperated roosters.

The average energy content of the low frequency

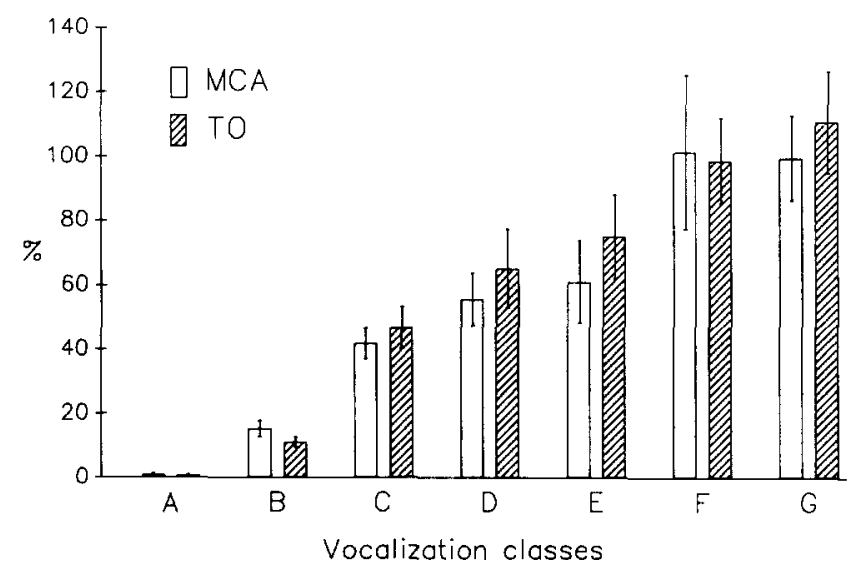

Fig. 6. EMG amplitude as a function of vocalization amplitude, expressed as classes of percentage of the maximal vocalization amplitude (A, 0-10\%; B, 10-20\%; C, 20-30\%; D, 30-40\%; E, $40-50 \% ; \mathrm{F}, 50-60 \%$; and $\mathrm{G}, 60-100 \%$ ), evoked in adult roosters by stimulating mesencephalic 'calling area' (MCA) and tractus occipitomesencephalicus (TO). The amplitude values are the area of full-wave rectified EMG and of vocalization activity. Mean of the recordings obtained in 10 fowl \pm S.E.M. 


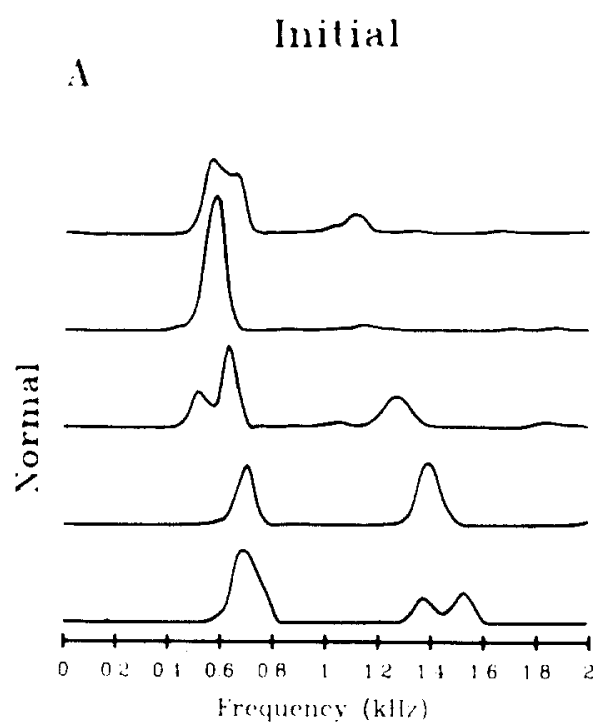

B

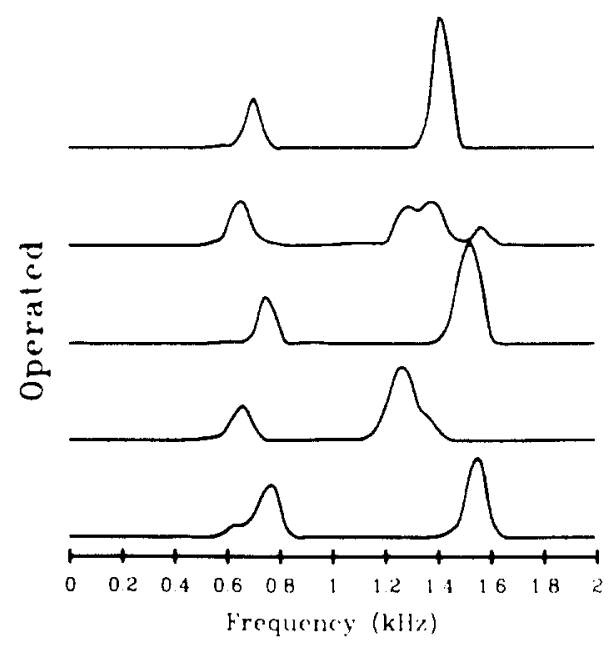

Intermediate

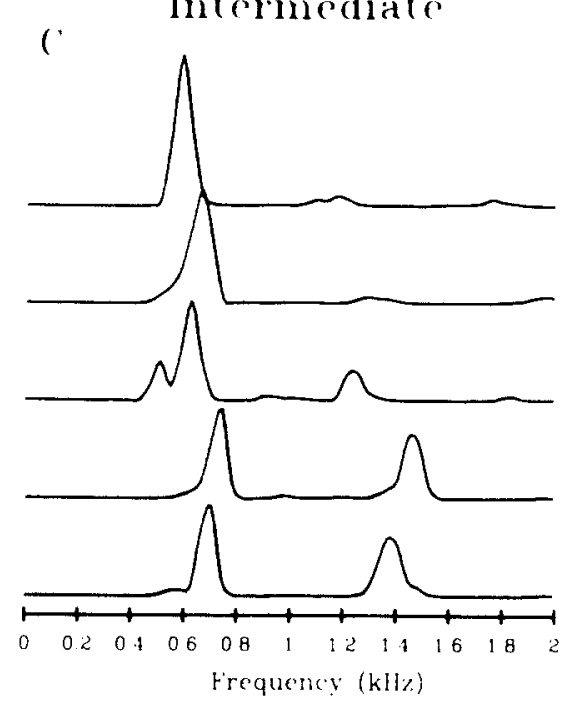

I)

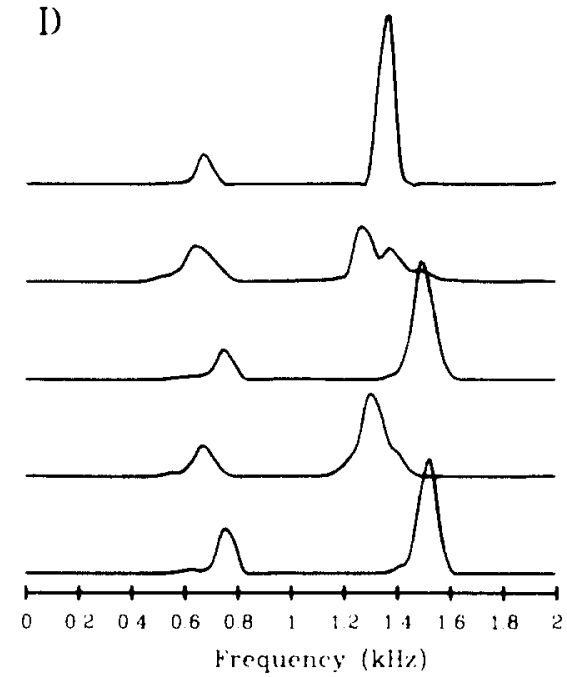

Final
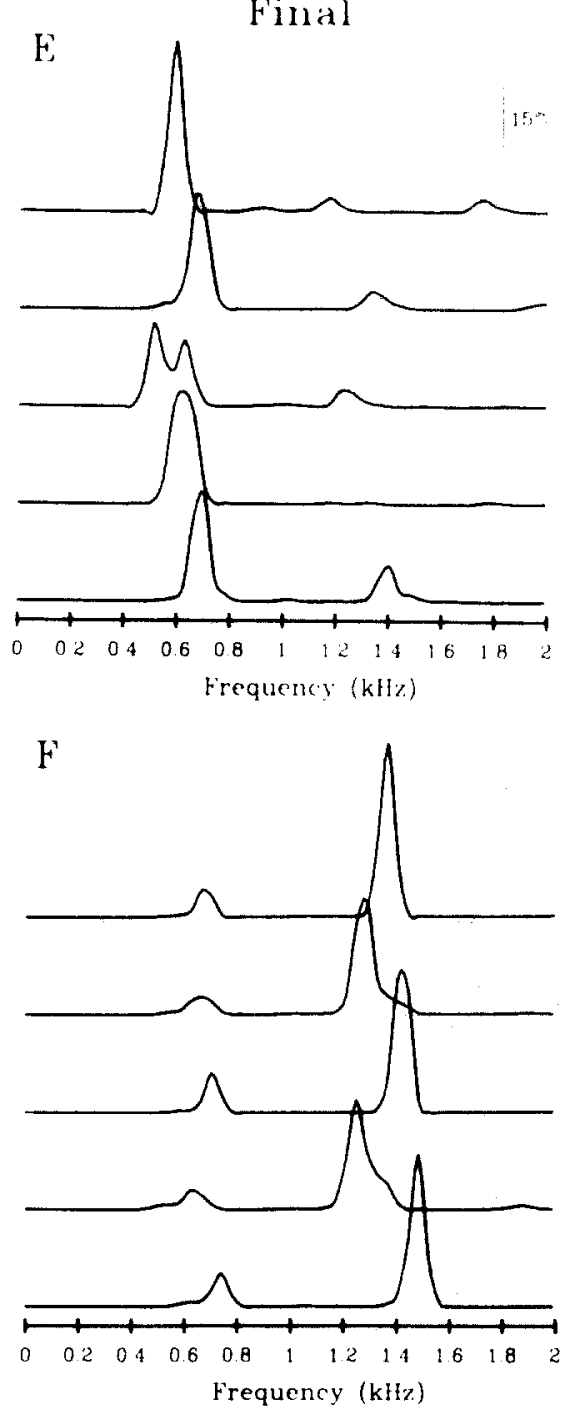

Fig. 7. Power spectra of crow of 5 normal (A, C, E) and 5 stapedectomized (B, D, F) roosters. In each rooster, the crow was analyzed at the time of its appearance (initial), some days later (intermediate) and when its pattern became stable (final).

component $(633 \mathrm{~Hz})$ in normal and sham operated roosters was $30.3 \pm 3.67 \%$ of the total energy when the crowing became stable, while that of the higher frequency component $(1300 \mathrm{~Hz})$ was $4.35 \pm 1.4 \%$. On the contrary, in operated roosters, when the crowing was stabilized, the low frequency component $(700 \mathrm{~Hz})$ showed an average energy content of $7.04 \pm 1.05 \%$, whereas the average energy content of the higher frequency component $(1400 \mathrm{~Hz})$ was $35.02 \pm 3.1 \%$.

The differences in the energy content of both crow components between normal and operated roosters were statistically significant (Mann-Whitney, $P<0.05$ ). The finding that no consistent difference in the acoustic structure of the crow, existed between normal and sham operated roosters, ruled out completely the possibility that the changes observed in stapedectomized roosters were due to the surgical procedure.

\section{DISCUSSION}

Our results show remarkable differences in the stapedius muscle activity associated with electrically induced vocalization in young and adult roosters.

Unlike young roosters, the stapedius muscle EMG response associated with vocalization in adult cocks always shows a higher threshold and a longer latency than that of vocalization. Moreover, its maximal amplitude is constantly lower than that detected in young roosters, despite the fact that the amplitude of the vocalization response is much higher.

More evidence was obtained by stimulating the occipitomesencephalicus tract in adult roosters, the major projection from the Archistriatum to mesencephalic 'calling area' ${ }^{17}$. A vocalization-related stapedius muscle EMG response showing the same characteristics as that 
elicited by stimulating the mesencephalic 'calling area' was observed. This finding rules out the possibility that, because of the enlargement of the mesencephalic 'calling area' in adulthood, the low vocalization-related stapedius muscle activity was due to the inability of electrical stimulation to activate a sufficient number of vocalization neurons to drive a higher stapedius muscle response. Furthermore, stapedius muscle activity alone, elicited in adult roosters ${ }^{9}$, was also reduced, and this finding was associated with a reduction in the size of mesencephalic loci, from which electrical stimulation could elicit the stapedius response. This observation suggests that the decrease in adult stapedius muscle activity could be due to a loss of mesencephalic stapedius controlling neurons, which could result from neuronal death. The shrinking of the stapedius controlling area can also account for the threshold and latency increase of the stapedius muscle response in adulthood. In fact in young roosters, when the direct activation of stapedius controlling neurons occurs, the latency of the stapedius muscle EMG response decreases, becoming shorter than that of the vocalization $^{8,9}$. In adult cocks, as a consequence of the presumed reduction in the number of stapedial neurons, only a secondary activation can probably take place at the stimulus intensities we used.

Our findings suggest that vocalization-related stapedius muscle activity is acutely reduced in adult roosters. These results, obtained during electrically induced vocalization, have to be validated by recording from awake birds, to confirm that the changes in stapedius muscle activity, detected in our experimental conditions, persist in awake spontaneously vocalizing birds. However, this seems to be likely considering that there is a datum in literature ${ }^{4}$ showing that stapedius muscle activity associated with spontaneous vocalization in young chickens is like that electrically induced.

Our findings question the hypothesis that the stapedius muscle only acts to protect the inner ear receptor cell during vocalization, as its activity appears markedly reduced just when the animal produces its loudest

\section{REFERENCES}

1 Borg, E. and Counter, A., The middle-ear muscle, Scient. Am., 261 (1989) 74-80.

2 Borg, E., Counter, S.A. and Rydquist, B., Contraction properties and functional morphology of the avian stapedius muscle, Acta Otolaryngol., 88 (1979) 20-26.

3 Borg, E., Counter, S.A. and Lännergren, J., Analysis of the avian middle ear muscle contraction by strain gauge and volume and impedance change measures, Comp. Biochem. Physiol., 71A (1982) 619-621.

4 Counter, S.A. and Borg, E., Physiological activation of the stapedius muscle in Gallus gallus, Acta Otolaryngol., 88 (1979) 13-19.

5 Counter, S.A. and Borg, E., The avian stapedius muscle. vocalizations. Hence, our findings suggest that stapedius muscle activity occurs during vocalization mostly in young fowl and may be involved in vocal development. A possible mechanism could be the modulation of the auditory feedback during such development, so that feedback occurs at a specified frequency and amplitude.

It has been suggested that song birds learn to sing only by hearing themselves vocalize and use the auditory feedback to match their voice with the intended pat$\operatorname{tern}^{11-15}$. This mechanism has been ruled out for the development of crowing in chicken ${ }^{10}$ which is thought to be completely independent of auditory input.

Our results demonstrate that strong differences exist in the crowing of normal and stapedectomized roosters, and so we suppose that auditory feedback can play a role in the development of adult vocalization also in this avian species. The modulatory action exerted by the stapedius muscle on auditory feedback, probably filters the acoustic input generated by the cock's own voice. When this filtering is not possible then perhaps sound production is modified until it generates the expected feedback that would have occurred with filtering. Recent work in an other avian species has shown that the energy of various harmonics of a same sound can be willfully controlled and imitated ${ }^{18}$ and this same control is apparently available to young roosters. As vocal development ends, so too ends most of the stapedial attenuation of self-generated vocal input.

It is conceivable that in adults the persistent low stapedius muscle activity is aimed at protecting the inner ear receptor cells against overstimulation by the bird's own voice as suggested ${ }^{1,4,5}$.

Work aimed at identifying the exact mechanism by which the stapedius muscle exerts its modulatory role during the different stages of the vocal development is in progress.

Acknowledgement. This work was supported in part by a grant from the Italian Ministry of Education.

Influence of auditory sensitivity and sound transmission, Acta Otolaryngol., 94 (1982) 267-274.

6 Counter, S.A., Borg, E. and Lännergren, J., Basic contraction properties of the avian stapedius muscle, Acta Physiol. Scand., 111 (1981) 105-108.

7 Grassi, S., Magni, F., Maurizi, M. and Ottaviani, F., $A$ Stereotaxic Atlas of the Brain of the Domestic Chick (Gallus gallus), Maggioli, Rimini, 1984.

8 Grassi, S., Magni, F. and Ottaviani, F., Mechanisms controlling vocalization-related stapedius muscle activity in chickens (Gallus gallus), J. Comp. Physiol., 162 (1988) 525-532.

9 Grassi, S., Ottaviani, F. and Bambagioni, D., Vocalization and stapedius muscle activity evoked by local electrical stimulation of midbrain in the chicken (Gallus gallus), Brain Research, 490 (1989) 345-349. 
10 Konishi, M., The role of auditory feedback in the vocal behavior of the domestic fowl, Z. Tierpsychol., 20 (1963) 349-367.

11 Konishi, M., Auditory environment and vocal development in birds. In R.D. Walk and H.L. Pick (Eds.), Perception and Experience, Plenum, New York, pp. 105-118.

12 Konishi, M., Birdsong: from behavior to neuron, Ann. Rev. Neurosci., 8 (1985) 125-170.

13 Marler, P. and Waser, M.S., The role of auditory feedback in canary song development, J. Comp. Physiol. Psychol., 91 (1977) $1-7$.

14 Marler, P. and Sherman, V., Structure in sparrow song without auditory feedback: an emendation of the auditory template hypothesis, J. Neurosci., 3 (1982) 517-531.
15 Nottebohm, F., The role of sensory feedback in the development of avian vocalizations, Proc. XIV Int. Ornithol. Congr., (1967) 265-280.

16 Oeckinghaus, $H$. and Schwartzkopff, J., Electrical and acoustical activation of the middle ear muscle in a songbird, J. Comp. Physiol., 150 (1983) 61-67.

17 Phillips, R.E., Youngren, O.M. and Peek, F.W., Repetitive vocalization evoked by local electrical stimulation of avian brains. I. Awake chickens (Gallus gallus), Anim. Behav., 20 (1972) 689-705.

18 Williams, H., Cynx, J. and Nottebohm, F., Timbre control in Zebra finch (Taeniopygia guttata) song syllables, J. Comp. Psychol., 103 (1989) 366-380. 